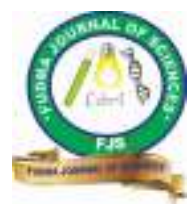

FUDMA Journal of Sciences (FJS)

ISSN online: $2616-1370$

ISSN print: 2645 - 2944

Vol. 4 No. 3, September, 2020, pp $731-734$

DOI: https://doi.org/10.33003/fjs-2020-0403-315

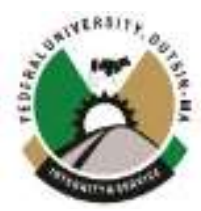

\title{
COMPARATIVE STUDY ON THE SYNTHESES OF CARBON NANOMATERIALS USING POLYETHYLENE AND RISK HUSK AS CARBON PRECURSOR.
}

\author{
${ }^{* 1}$ Kure, N., ${ }^{1}$ Daniel, I. H., ${ }^{1}$ Machu, B. U., ${ }^{2}$ Bello, I. A. and ${ }^{3}$ Asnawi, M. \\ ${ }^{1}$ Department of Physics, Kaduna State University, Kaduna State, Nigeria. \\ ${ }^{2}$ Division of Agricultural Colleges, Ahmadu Bello University Zaria, Kaduna State, Nigeria. \\ ${ }^{3}$ Institute of Technology, Universiti Putra Malaysia, Malaysia. \\ *Corresponding Author's Email: kurenicodemus@gmail.com
}

\begin{abstract}
The study compares the syntheses of carbon nanostructures (CNS) using polyethylene (PE) and Rice husk (RH) as carbon precursor via commercial microwave oven at $2.45 \mathrm{GHz}$. The Microwave energy offers the requisite temperature for catalytic disintegration of the carbon precursors at $750{ }^{\circ} \mathrm{C}$ under atmospheric pressure. The CNS were grown on coated silicon dioxide. The as-synthesized CNS was analysed with Raman spectroscopy which shows carbon quality was found to be 0.92 and 1.01 in PE and RH respectively, indicating good graphitic nature with average diameter at (16.0 to 20.0$) \pm 0.5 \mathrm{~nm}$. The high intensity ratio is attributed to the defect mode in the CNS. The Field Emission Scanning Microscope (FESEM) analysis shows a warped and randomly oriented structures with an interlayer spacing of about $0.35 \mathrm{~nm}$ in the internal structure of most CNS. Furthermore, the level of purity in the graphitic nature of the CNS were obtained with Thermogravimetric Analysis (TGA) technique with $90 \%$ in PE and $50 \%$ in RH. Hence, a fast and cheaper method of synthesizing CNS utilizing microwave energy was demonstrated at $750{ }^{\circ} \mathrm{C}$ under atmospheric pressure. Lastly, the presence of catalyst, carbon precursors and plasma are necessary for the microwave heating and synthesis process.
\end{abstract}

Keywords: Carbon nanostructures, Raman, Rice Husk, Polyethylene, Silicon Substrate.

\section{INTRODUCTION}

The emergence of nanotechnology has attracted much interest from scientific community in the area of carbon-based science due its exceptional carbon-carbon bonding assists carbon to form its allotropes, amongst such carbon nanostructures (CNS) are carbon nanofibers, graphites, graphenes, and carbon nanotubes (Kroto, 1991; O'Connell, 2006; Kure et al., 2015; Kure et al., 2017). Their exceptional properties such as electrical, thermal, chemical and mechanical properties attract interest from researchers (Kroto et al. 1991; Iijima, 1991). Extensive research has been carried out on how to synthesize this carbon-based nanomaterial. Among such methods are arc discharge (Iijima, 1991), laser ablation (Guo et al. 1995), chemical vapor deposition (Suriani et al. 2013) and Microwaveassisted chemical vapor deposition (Kure et al., 2017), with Carbon precursor playing a vital roles in the formation of CNS (Kure et al., 2015 and Kure et al., 2017). These techniques of synthesizing CNS are believed to be time consuming and expensive. In order to reduce cost and time wastage, an attempt was made to synthesize CNS using polyethylene (PE) and rice husk $(\mathrm{RH})$ as carbon precursor via $600 \mathrm{~W}$ household microwave oven at $2.45 \mathrm{GHz}$ (Kure et al., 2017 and Muhammad et al., 2018). The PE and RH has dielectric loss tangent at $2.45 \mathrm{GHz}$, which makes them a good conducting polymer (Hotta et al. 2011; Kure et al., 2017). The synthesis utilizes microwave heating due to its advantages over the conventional methods such as rapid reaction time and volumetric heating which provides the temperature needed for catalytic decomposition of PE and RH (Bajpai et al., 2014; Kure et al., 2017 and Muhammad et al., 2018).

\section{MATERIALS AND METHODS}

A tubular quartz tube was used as reaction chamber. The household microwave oven was adapted to introduce the tubular quartz duct across it. It was vacuum below 0.81 mbar of atmospheric pressure via rotary vacuum pump continually throughout the synthesis process. Polyethylene beads of $100 \mathrm{mg}$ were used as carbon sources and silicon dioxide as substrate. Iron (III) nitrate was impregnated on the substrate which served as the trigger material for CNS growth. The catalyst and carbon source were sited within the reaction chamber. The temperature of the process was measured using thermocouple attach to Fluke multimeter to be $750{ }^{\circ} \mathrm{C}$ (Kure et al, 2017). This technique utilizes the advantage of microwave heating over conventional heating, to synthesize the CNS at much faster rate. The as-grown nanomaterial was allowed to reach ambient temperature before characterization. Raman spectroscopy was used to analyse the carbon quality using Witec alpha 300R with $532 \mathrm{~nm}$ laser excitation wavelength. Field emission scanning electron microscope (FESEM) images were produced via Joel JSM$7600 \mathrm{~F}$ to know the structural morphology. A Mettler thermobalance TG-50 in a Mettler TA-4000 System with temperature ranging from $30{ }^{\circ} \mathrm{C}$ to $1000{ }^{\circ} \mathrm{C}$ with interval of 10 ${ }^{\circ} \mathrm{C} / \mathrm{min}$ under oxygen gas with flow rate of $50 \mathrm{~mL} / \mathrm{min}$ was used to carryout thermogravimetric analysis (TGA) in order to determine the level of purity in the CNS. The diameter distribution of the CNS was determine using Image J processing software.

Figure 1 depicts the experimental setup used for the derivation of carbon nanomaterials from the carbon precursors. The synthesizing pressure plays a vital in synthesis process, hence, the utilization of microwave energy. The PE pellets and RH 
powders were irradiated for 4 minutes and 38 minutes respectively (Kure et al., 2017 and Muhammad et al., 2018). The temperature and pressure were maintained at $750^{\circ} \mathrm{C}$ and $3 \times 10^{\circ}$ mbar respectively for the both carbon precursors (Kure et al., 2017 and Muhammad et al., 2018).

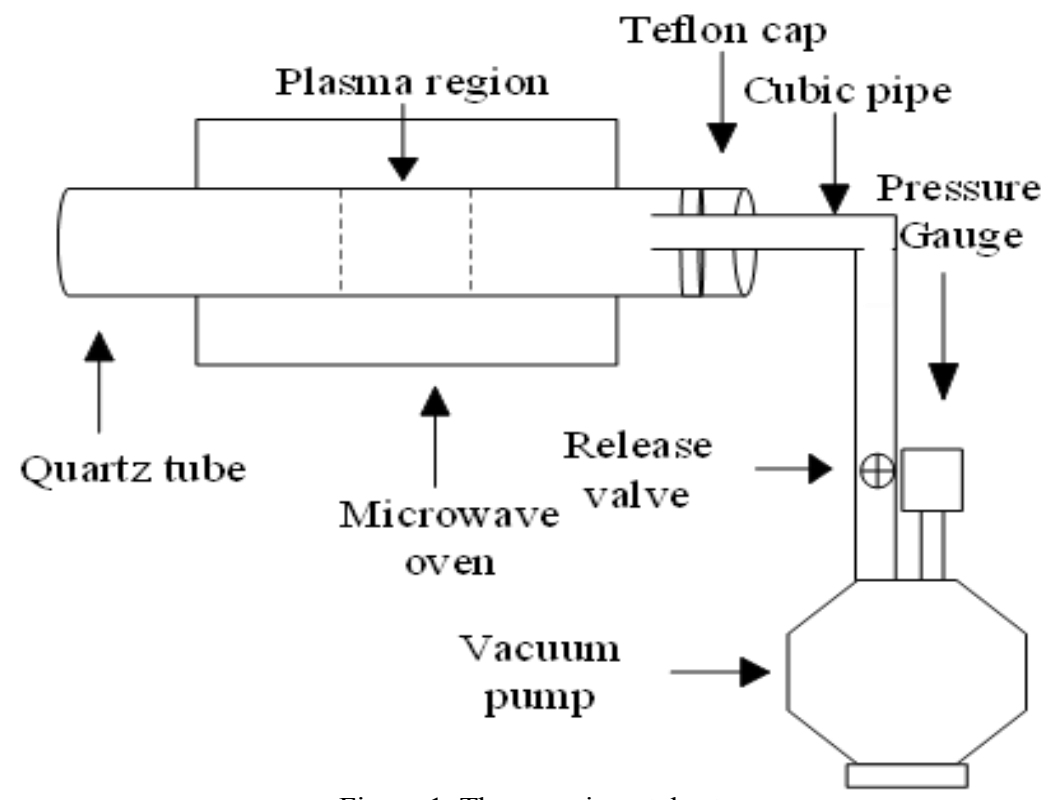

Figure 1. The experimental setup

\section{RESULTS AND DISCUSSIONS}

Results obtained are as depicted in Figure 2 (a) and (b) which shows the Raman spectrum of the carbon nanostructures from carbon precursors (PE and $\mathrm{RH}$ ) with two protruding $\mathrm{D}$ and $\mathrm{G}$ peaks respectively. Figure 2 (a) shows two protruding peaks at $1336.77 \mathrm{~cm}^{-1}$ and $1588.50 \mathrm{~cm}^{-1}$ indicating defect (D) and graphite $(\mathrm{G})$ mode respectively. The defect mode indicates the disordered carbon atom and graphite mode indicates the ordered carbon atoms on the CNS (Kure et al., 2017). The ratio of the defect intensity (ID) and graphite intensity (IG) is to determine the CNS carbon quality and it was calculated to be 0.97 . The defect mode increases as a result of amorphous carbon indicating impurity (Kure et al., 2015). From Figure 2 (b), D peak was observed at $1335 \mathrm{~cm}^{-1}$. The induced peak shows defective graphitic structures associated with $\mathrm{sp}^{3}$ honeycomb carbon atom network. The $G$ peak observed at $1601 \mathrm{~cm}^{-1}$ indicates the level of order in $\mathrm{sp}^{2}$ honeycomb carbon atom network (Muhammad et al., 2018). The ratio of $\mathrm{D}$ band to $\mathrm{G}$ band $(I \mathrm{D} / I \mathrm{G})$ was used to estimate the degree of defects present on the carbon nanostructures. The ratio of $(I \mathrm{D} / I \mathrm{G})$ for the assynthesized nanostructures was calculated to be 1.01 (Muhammad et al., 2018). The peaks intensities ( $I \mathrm{D} / I \mathrm{G})$ shows lower quality of $\mathrm{sp}^{2}$ carbon structures due to amorphous carbon and structural defects in carbon nanomaterials (Muhammad et al., 2018). 

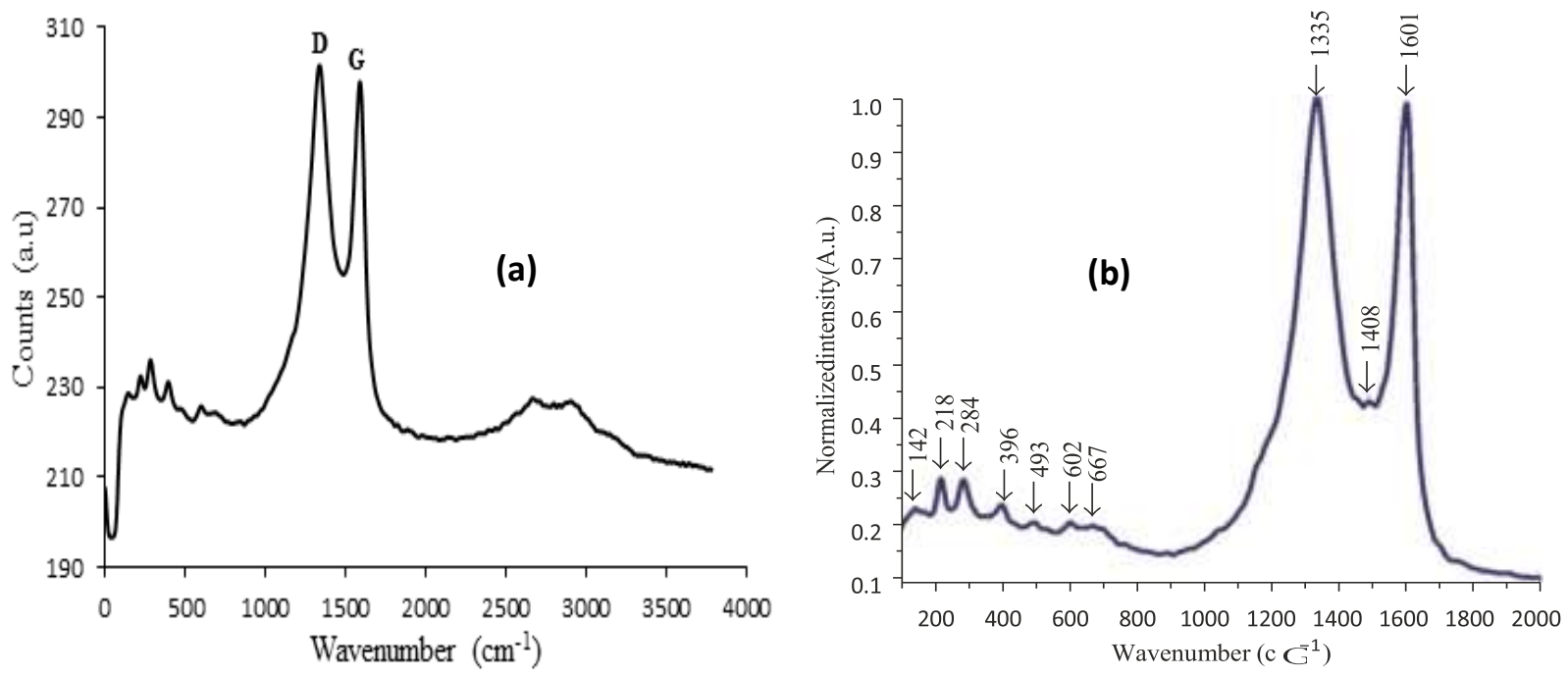

Figure 2(a) Raman spectrum of PE (b) Raman Spectrum of RH

Figure 3(a) and (b) shows the surface morphology of the derived CNS. These images from FESEM shows the formation of a twisted and flower-like CNS with several micrometers longdue to catalytic decomposition of the carbon precursors. The CNS size variations may be attributed to defect mode as depicted in
Figure 3 with diameter range between (16.0 to 20.0) $\pm 0.5 \mathrm{~nm}$. From Figure 3, it shows a bright-tip color on the CNS indicating the presence of the catalyst remained (Kure et al., 2015; Kure et al., 2017 and Muhammad et al., 2018).
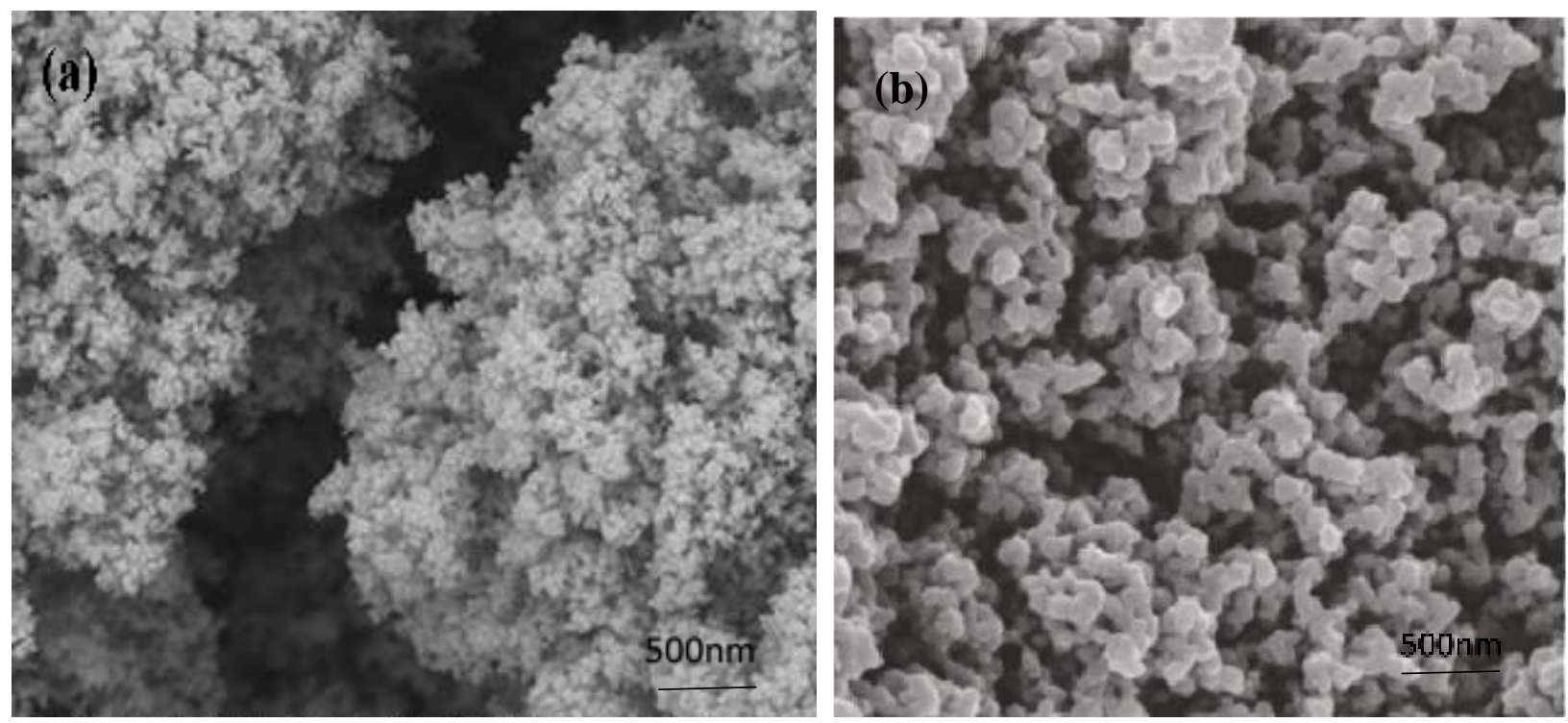

Figure 3 (a) FESEM image of PE CNS (b) FESEM image of RH CNS

The TGA results were shown in Figure 4 (a) and (b) below. From Figure 4 (a), it shows a quick oxidation of CNS from $540{ }^{\circ} \mathrm{C}$ to $640{ }^{\circ} \mathrm{C}$ with $90 \%$ sample weight loss; the sharp peak observed from DTGA result corresponds to this region. At $640{ }^{\circ} \mathrm{C}$ the sample contains $2 \%$ residue which continues to drop gradually to $1.3 \%$ at $1000{ }^{\circ} \mathrm{C}$ (Kure et al., 2017). The TGA result shown in Figure 4 (b) shows $10 \mathrm{wt} \%$ weight reduction at $343{ }^{\circ} \mathrm{C}$ due to the presence of amorphous carbons and structural defects which further confirms the Raman analysis as depicted in Figure 2(a) and (b); a rapid decomposition of the sample was observed from
$90 \mathrm{wt} \%$ to $4.6 \mathrm{wt} \%$ within the temperature range of $343{ }^{\circ} \mathrm{C}$ to $490{ }^{\circ} \mathrm{C}$ respectively; thus, this suggests the presence of carbon nanomaterials (Muhammad et al., 2018). The DTGA analysis showed a major protruding peak at $421.5{ }^{\circ} \mathrm{C}$ with $50 \mathrm{wt} \%$ loss which falls below what is required for CNTs when compared with previous results obtained by other research groups. However, this result is justifiable since the presence of iron oxide in CNS sample could result in lower decomposition temperature (Muhammad et al., 2018). 


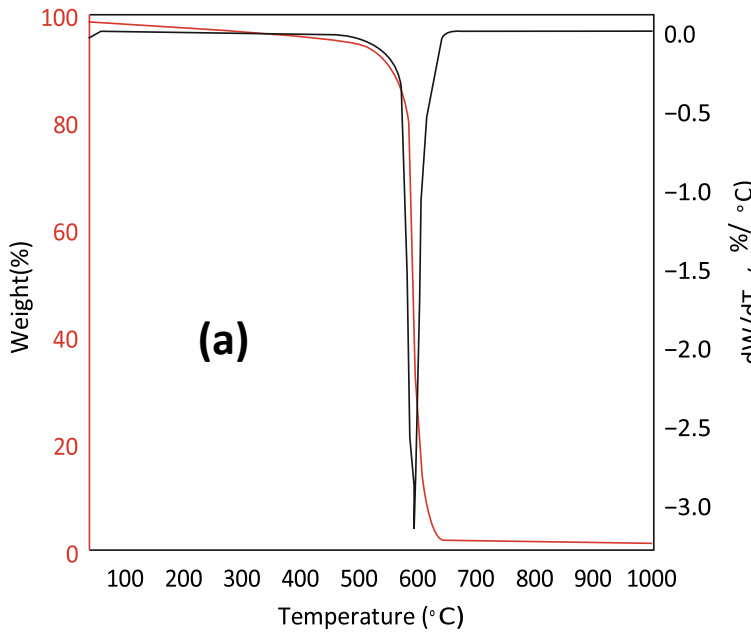

Figure 4 (a) TGA result of PE CNS

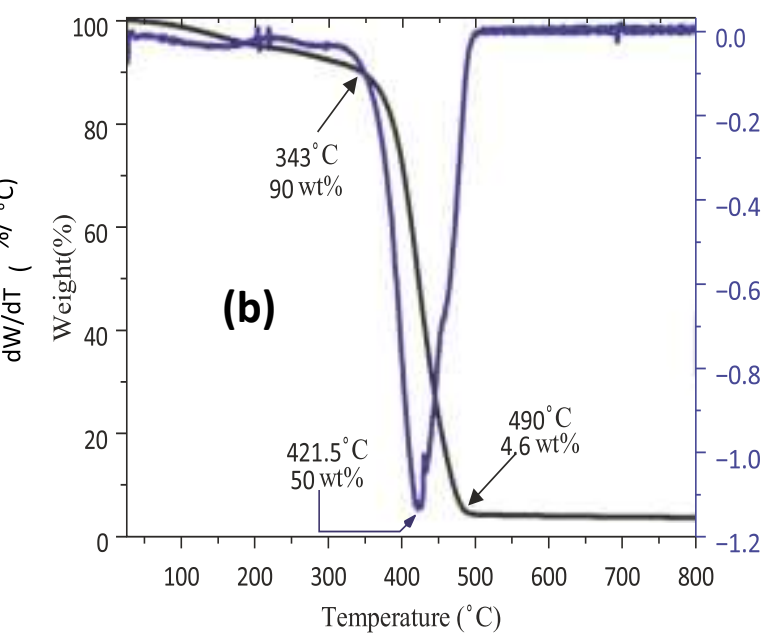

(b) TGA result of RH CNS

\section{CONCLUSION}

This study shows that, the alternative technique of synthesizing CNS via Microwave-Assisted PECVD at the temperature of 750 ${ }^{\circ} \mathrm{C}$, is more economical and faster which depend on the nature of the carbon precursors susceptibility to microwave irradiation. Hence, the time duration of CNS derived from PE is at 4 minutes and $\mathrm{RH}$ at 38 minutes. The CNS carbon quality was found to be 0.97 and 1.01 for PE and RH respectively, FESEM shows flower-like structures of average diameter size to be (16.0 to $20.0) \pm 0.5 \mathrm{~nm}$ for the both carbon precursors. The carbon purity was $90 \%$ to $50 \%$ for PE and RH respectively. CNS may be produced with high yield and purity if parameters are properly control.

\section{REFERENCES}

Bajpai, R., Wagner, H. D. (2014). Fast growth of carbon nanotubes using a microwave oven. Carbon, 82, 327-336.

Guo, T., Nikolaev, P., Rinzler, A. G., Tombnek, D., Colbert, D. T., Smalley, R. E. (1995). Self-Assembly of Tubular Fullerenes. J. Phys. Chem, 99, 10694-10697.

Hotta, M., Hayashi, M., Lanagan, M. T., Agrawal, D. K., Nagata, K. (2011). Complex Permittivity of Graphite, Carbon Black and Coal Powders in the Ranges of X-band Frequencies (8.2 to 12.4 $\mathrm{GHz}$ ) and between 1 and $10 \mathrm{GHz}$. ISIJ International, 51(11), 1766-1772.

Iijima, S. (1991). Helical microtubules of graphitic carbon. Letters of Nature, 354, 56-58.

Kroto, H. W., Allaf, A. W., Balm, S. P.. (1991). C60 Buckminsterfullerene. Chemical Review, 91(6), 1213-1235.
Kure, N., Hamidon, N. M., Azhari, S., Usman I. K., Hasan, I. H., Ismail, I. (2015). Synthesis of Carbon Nanotubes Using Microwave Oven. IEEE Explore.

Kure, N., Hamidon, N. M., Maryam, I. M., Yusoff, H. M., Shuhazlly, N. M., Azhari, S., Yunusa, Z. (2017). Simple Microwave-Assisted Synthesis of Carbon Nanotubes Using Polyethylene as Carbon Precursor. Journal of Nanomaterials. 2017, Article ID 2474267, 1-4.

Kure, N., Hamidon, N. M., Daniel, I. H., Kassimu, A. A., Sarki, S. H. (2018). Construction of Plasma Enhanced Chemical Vapor Deposition Technique via Commercial Microwave Oven. American Association for Science and Technology, 5(2), 46-49.

Muhammad, A., Saman A., Hamidon, H. M., Ismayadi, I., and Intan, I. (2018). Synthesis of Carbon Nanomaterials from Rice Husk via Microwave Oven. Journal of Nanomaterials, 2018, Article ID 2898326, 1-5

O'Connell, M. J. (2006). Carbon Nanotubes Properties and Applications. Taylor \& Francis group, Boca Raton, Flourida. Suriani, A. B., Dalila, A. R., Mohamed, A., Mamat, M. H., Salina, M., Rosmi, M. S., Rosly, J., Md Nor, R., Rusop, M. (2013). Vertically aligned carbon nanotubes synthesized from waste chicken fat. Materials Letters, 101, 61-64. International license viewed via https://creativecommons.org/licenses/by/4.0/ which permits unrestricted use, distribution, and reproduction in any medium, provided the original work is cited appropriately. 